\section{The misperception of angles: Estimating the vertex of converging line segments*}

\author{
DANIEL J. WEINTRAUB $\dagger$ and VEIJO VIRSU \\ University of Michigan, Ann Arbor, Michigan 48104
}

Estimates of the point of intersection of converging line segments depended upon the angle between lines and the orientation of the display. Main conclusion: The tilt of a line is perceptually altered to appear more nearly parallel to the more closely aligned axis, either horizontal or vertical, of an O's visual field.

A characteristic of many geometrical illusion figures is that they contain converging straight lines. There is a notable lack of consensus concerning the explanation of such illusions. One pervasive descriptive generalization (Luckiesh, 1922; Wallace, 1966) is that acute angles tend to be overestimated. For example, in the Poggendorff illusion (illustrated in Fig. 2) each segment of the diagonal is said to be rotated toward the horizontal because acute angles are perceived as too large. The perceptual rotation explains why the segments do not appear collinear. Chiang (1968) has revived the theory that, as a result of optical aberrations in the eye, vertices of angles are blurred on the retina. As a consequence of blurring, the perceived intersection of the lines is displaced inward providing an explanation for the overestimation of acute angles. At least two studies (Hotopf, 1966; Restle, 1969) have, however, cast doubt upon the acute-angle overestimation hypothesis as a satisfactory account of the Poggendorff illusion.

Gregory (e.g., 1966, 1968) has advocated a misapplied-constancy theory to explain illusions with converging lines. Since converging lines in a two-dimensional display provide the same retinal image as parallel lines proceeding away from the $\mathrm{O}$ in his three-dimensional world, the lines constitute a powerful cue for distance. Though the $O$ does not necessarily experience the flat display in three dimensions, he nonetheless uses such a cue in experiencing size features associated with the display. Thus, the $\mathrm{O}$ is presumed to misapply the principles of constancy, interpreting retinal information to give an experience inappropriate to the

*Supported by a United States Public Health Service Research Scientist Development Award (K 3-MH-35.253) and by National Science l oundation (irant GB 8181 to D.J.W., and by Kamarincuvos H. Rosenberg's travel grant from the University of Helsinki to V.V. We thank I illian Tong for serving as experimenter.

t Address: Department of Psychology, University of Michigan, Ann Arbor. Michigan 48104. two-dimensional display. For example, in the Ponzo illusion (illustrated in Fig. 2) the overestimation of the length of the upper horizontal line segment is accounted for by assuming that size-constancy tendencies are activated by the converging pair of lines, and consequently the upper horizontal line segment is perceived as larger, though both retinal size. (If two objects at different physical distances produce the same retinal size, then the farther one must be physically larger.) But size-constancy tendencies should be acting also upon the converging lines, perceptually enlarging the narrowing physical distance between them. A misapplied-constancy explanation rooted in parallelism implies, then, that all angles should be underestimated because line convergence will always tend to bc discounted psychologically as parallel lines receding in space.

A second interpretation of the misapplied-constancy hypothesis holds that converging lines might be interpreted perceptually as a rectangular corner (Gregory, 1968) rather than as a pair of parallel lines. For example, with the converging lines of the Ponzo illusion, the $O$ can be considered to be facing an inside corner. If the angular separation of the lines exceeds $90 \mathrm{deg}$, the tendency to perceive the corner as rectangular should induce underestimation of the angle. However, one should not conclude that all angles less than $90 \mathrm{deg}$ should be overestimated. The rectangular-corner version of the misapplied-constancy hypothesis is, in fact, inapplicable to an acute-angle Ponzo example because no right angle when viewed from inside can be imaged upon the retina at less than $90 \mathrm{deg}$. In addition, certain viewing positions "outside" a right angle, namely, facing the imaginary angle formed by extending the sides of the original angle through its own vertex, will also have a retinal representation greater than 90 deg.

Utilizing a suggestion by Virsu for a major simplification of a converging-line display, we presented Os with a pair of converging line segments and asked them horizontal line segments produce the same to determine the point of intersection. Errors in locating the intersection can be interpreted as misperceptions of the angle formed by the lines. Because the lines themselves do not form an intersection, "blurredness" (Chiang, 1968) or neural inhibition (von Békésy, 1967) in the region of the intersection should not complicate the results.

\section{METHOD}

Two experiments were conducted varying the angular separation between the converging line segments. In Experiment 1, the display was presented symmetrically with vertex pointing upward with respect to O's visual field. In Experiment 2, the display was asymmetrical with respect to the vertical axis of $O$ 's visual field; the left line segment was always vertical.

Experiment 1 consisted of four different data-collection phases. The initial phase on 24 Os using a set of six angular separations provided the impetus for the second phase on 24 new Os with an expanded set of 11 angles from $5-5 / 8$ to $168-3 / 4 \mathrm{deg}$. The third phase with 24 new participants, a supplement to the first phase, brought the number of observations on each of the 11 displays to 48 . In the fourth phase an additional 31 observations (again with new Os) were gathered on angles $<60 \mathrm{deg}$. Hence, there were judgments collected from 79 Os for each angle $<60 \mathrm{deg}$ and judgments from 480 os for angles $>60 \mathrm{deg}$ in Experiment 1. The data of Experiment 2 were gathered during the second phase of Experiment 1 so that, in addition to the symmetrical displays, 24 judgments were collected for each of 11 asymmetrical displays. ${ }^{1}$ The Os were students and staff at the University of Michigan.

Stimuli were drawn with black India ink on $22.9 \times 30.5 \mathrm{~cm}(9 \times 12$ in. $)$ white drawing paper. Two line segments, $30 \mathrm{~mm}$ long and $0.25 \mathrm{~mm}$ wide, were drawn with the true point of intersection $60 \mathrm{~mm}$ from the ends of the segments. Each pair of lines was drawn so that the true intersection was randomly displaced from the center of the paper. A large (diameter approximately $28 \mathrm{~cm}$ ) circular piece of transparent acetate with an irregular edge contained a black dot $(1.5-\mathrm{mm}$ diam) near the center. The acetate was used as an overlay, the dot denoting the intersection of the lines. The $O$, standing at the edge of the table, leaned over to peer down (approximately $50 \mathrm{~cm}$ from eye to paper) at the display, with his line of sight perpendicular to the display near the true point of intersection of the two line segments. He was told, "I would like you to place the dot at the point where the two lines would intersect if they were extended." The $O$ was asked not to tilt his head; he could move the dot in any 


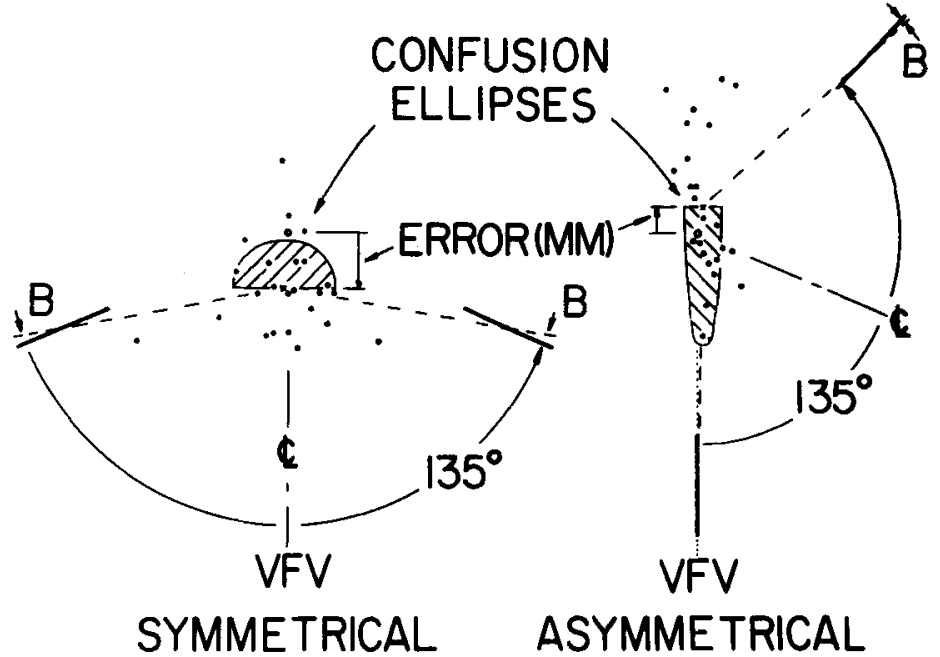

way desired. In any experiment, the angles were presented once to each. $O$ with different randomly determined orders for each $\mathrm{O}$.

The centerline (CL) refers to the bisector of the angle formed by the line segments (see Fig. 1). The visual-field vertical (VFV) refers to the line at the surface of the display which vertically bisects $O$ 's visual field. Thus, in Experiment 1 (symmetrical display) CL and VFV coincided, and in Experiment 2 (asymmetrical display) the left line segment and VFV coincided. The long edges of the display paper were always parallel to the VFV.

\section{RESULTS AND DISCUSSION}

The bidimensional deviations of the judgments were recorded in millimeters relative to the physically correct point of intersection. First, considerable illusion was present with many displays. For instance, with symmetrical displays, $73 \%$ of the Os placed the dot too high at $22 \frac{1}{2} \mathrm{deg}$ of angular separation and almost all Os (98\%) placed the dot too low at a $112 \frac{1}{2}$-deg angle. Second, two-dimensional scatterplots of the data disclosed that judgments were always distributed symmetrically about a vertical axis (i.e., parallel to the VFV) regardless of the location of the $\mathrm{CL}$ of the display.

Figure 1 depicts the symmetrical and asymmetrical displays for the angular separation of $135 \mathrm{deg}$. The diagrams include, in exaggerated scale, raw data points for the 24 Os of Phase 2 who judged both the symmetrical and the asymmetrical displays. The points show how each data distribution is oriented relative to the pair of converging lines. Figure 1 also depicts
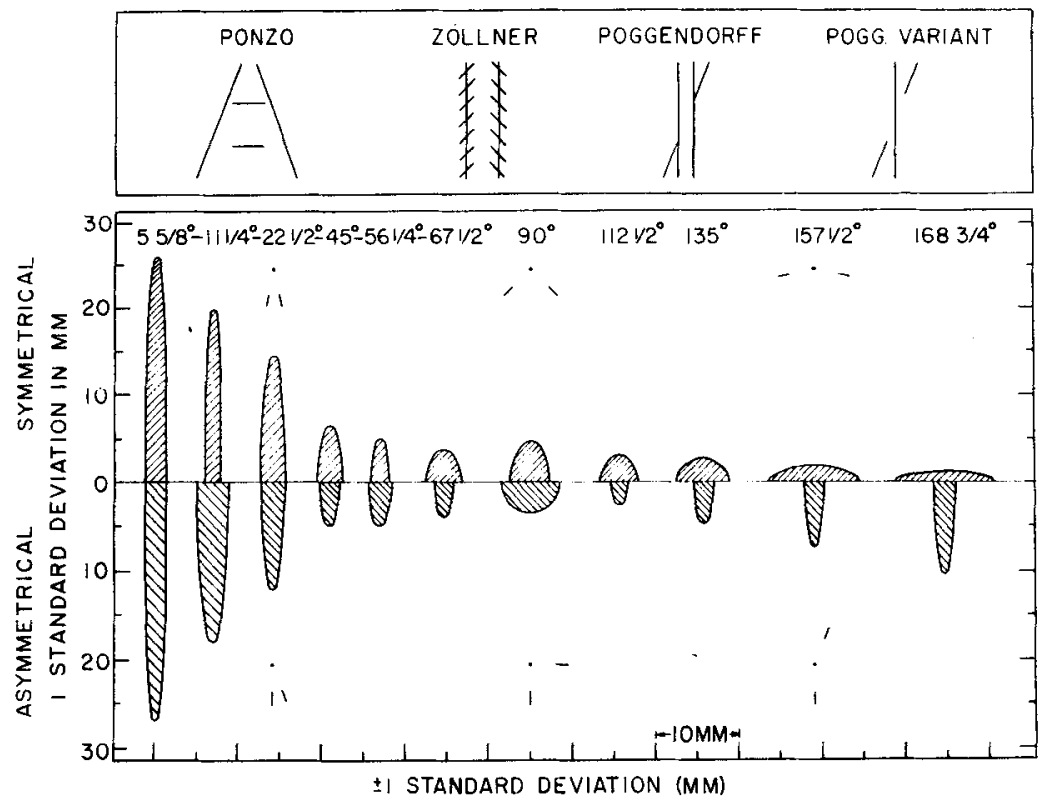

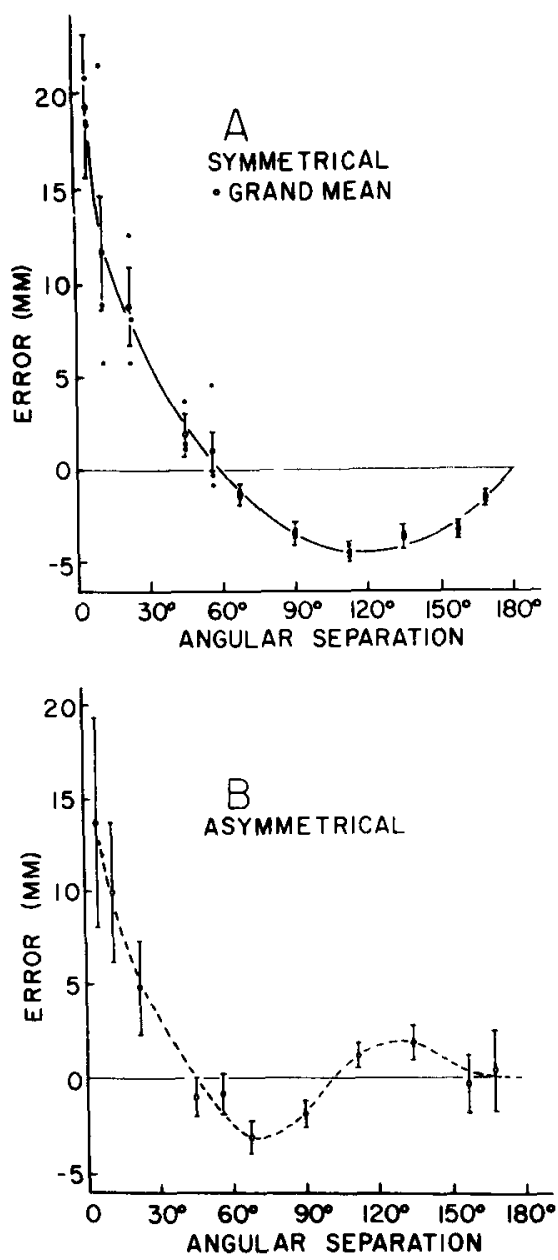

Fig. 3. Mean vertical error in determining the point of intersection of converging line segments as a function of their angular separation. \pm 1 standard error of the mean is shown by a vertical bar. A: Error for symmetrical displays. Filled circles show means for each data-collection phase. B: Error for asymmetrical displays.

along the centerline. Therefore, errors were measured in both experiments as vertical deviations with respect to the VFV, establishing the true point of intersection of the line segments as origin. Figure 3 presents the error means as vertical deviations in millimeters from the true point of intersection. The error functions for symmetrical and asymmetrical displays do not coincide. In symmetrical displays (Fig. 3A) the point was placed too high, implying underestimation of the angle between line segments for angular separations smaller than $60 \mathrm{deg}$, and too low, implying angle overestimation, for angular separations greater than $60 \mathrm{deg}$. On the other hand, for asymmetrical displays (Fig. 3B) the point was placed too low with angular separations in the range of $40.100 \mathrm{deg}$. Deviations of the means laterally about the VFV were small and random except for asymmetrical displays with angular separations exceeding $60 \mathrm{deg}$ that gave the following deviations of means to the right of the VFV: $671 \frac{1}{2} \mathrm{deg}=$ $1.17 \mathrm{~mm} *, 90 \mathrm{deg}=1.19 \mathrm{~mm}, 112 \frac{1}{2} \mathrm{deg}=$ $.70 \mathrm{~mm}^{*}, 135 \mathrm{deg}=.45 \mathrm{~mm}, 1571 / 2 \mathrm{deg}=$ $.50 \mathrm{~mm}^{*}, 168^{3 / 4} \mathrm{deg}=1.19 \mathrm{~mm} *$, starred values significantly different from zero at $\alpha=.05$.

The judgments may be interpreted, not only as displacements of the point of intersection, but also as errors in perceiving the inclinations of line segments. In fact, the error can be measured in degrees of tilt of a line segment as directly as it can be measured as a millimeter displacement of the intersection. A tilt measurement tends to represent the data more fairly since lateral as well as vertical deviations in the judged point of intersection contribute to a tilt error. The mean data in Cartesian coordinates for each illusion display were converted to a tilt error in degrees for every line segment, assuming a line segment to be tilted perceptually about its own midpoint (Angle B of Fig. 1). Figure 4 depicts tilt errors as a function of the orientation of a line segment with respect to the visual field vertical. Since in asymmetrical displays left line segments were oriented vertically, these 11 data points should have been plotted at zero deviation from VFV along the abscissa. However, each of the 11 was assigned the same abscissa value as its nonvertical right-line mate to facilitate pairwise comparisons of errors.

Note from Fig. 4 that, for displays symmetrical about the VFV, tilt errors are alike in magnitude but opposite in direction for each line of a pair. In asymmetrical displays, tilt errors are not at all the same for each line of a pair. Significantly, the right line segment, i.e., the tilted segment, follows approximately the same course of perceptual tilt as the right line segment of the symmetrical display. The left line segment, at all times vertical, shows a near-zero error or a moderate clockwise error. Thus, deviation of any line from the VFV is a far better description of judgmental error than either angular separation of line segments or orientation of the display. The data point again to a phenomenon that is largely independent of the angle between the lines but that appears to be related to the cardinal directions of the visual field.

The data of Fig. 4 conform approximately to the following hypothesis: Os reduce the angular disparity between a line segment and the nearer (in degrees of inclination) cardinal viewing axis. That is, tilted lines tend to appear as either more horizontal or more vertical with respect to an $\mathrm{O}$ 's visual field than they actually are

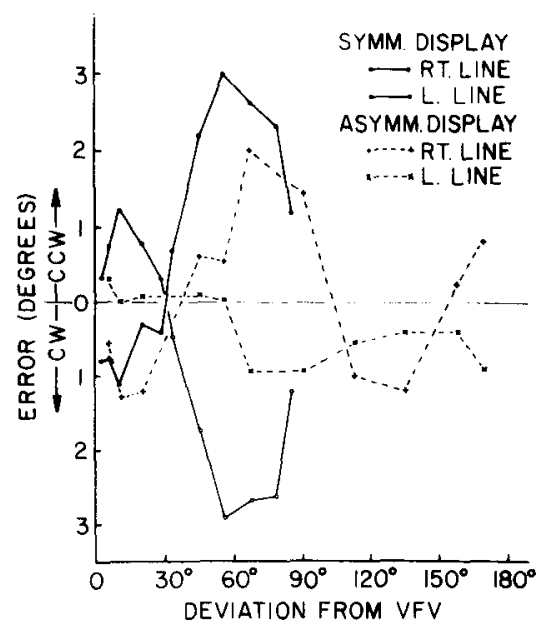

Fig. 4. Errors expressed as misperceived tilt. The left line of each asymmetrical display had zero deviation from VFV but was assigned the same abscissa value as its nonvertical mate.

When a line segment of either type of display is oriented near the VFV, it is tilted perceptually toward the vertical. When a line segment is oriented more nearly horizontally, it assimilates (is perceptually tilted toward) the horizontal. In symmetrical displays angles greater than $168^{3 / 4} \mathrm{deg}$ were not used; consequently, deviations from VFV do not exceed half that angle. But the tilt errors derived from asymmetrical displays behave properly even beyond $90 \mathrm{deg}$. As the right line exceeds $90 \mathrm{deg}$ deviation from VFV, tilt errors reverse to clockwise (assimilation toward horizontal). As the right line approaches vertical again $(180 \mathrm{deg})$ at the top of a display, tilt errors revert back to counterclockwise (assimilation toward vertical).

Interpreting the illusion as an error in perceived inclination of each line of a pair of segments permits a synthesis of the data of symmetrical and asymmetrical orientations. When the results are interpreted as millimeter displacements of the intersection, as in Fig. 3, there are clear differences between asymmetrical and symmetrical displays. However, when the errors are considered as perceptual inclinations of single line segments and the angle between the line segments is ignored, as in Fig. 4, line segments tilted the same amount with respect to the VFV show similar errors that can be interpreted as perceptual tilts toward the nearer cardinal axis of the visual field.

Assimilation toward a cardinal reference axis is not a sufficient explanation for the illusion. For example, our hypothesis implies that zero perceptual tilt error should obtain for a vertical or horizontal line. However, for the left lines of 
asymmetrical displays, i.e., the vertical-line data marked as crosses (xs) in Fig. 4, 6 of the 11 displays produced a substantial clockwise error. Second, the right line segment of the asymmetrical display showed a tilt error when that line was horizontal (deviation from VFV $=90 \mathrm{deg}$ ). These outcomes suggest that there is a factor related to the angular separation between the line segments that interacts with the assimilation toward a cardinal reference axis. The hypothesis of assimilation toward the cardinal axes is not accurate in detail, but it describes satisfactorily the main features of the results for both orientations of displays.

The present data do not permit an evaluation of the role of the horizontal-vertical borders of the paper on which the line segments were drawn. However, in perception the notion of cardinal viewing axes is an old and important one. For example, results of Witkin (1949) and Hofmann and Bielschowsky (1909) have shown that tilting a visual display or an O's body leads to an assimilative inclination of the subjective upright or horizontal. It is to be expected that insofar as the borders of the display affect subjective horizontal-vertical axes, the borders should exert an interactive influence upon the illusion itself.

\section{EPILOGUE}

Evidence suggests one strong factor as an explanation of the observed errors in judging the intersection of line segments, namely, misperception of tilt as a result of assimilation toward a psychological reference axis. Green and Hoyle (1964) have proposed a similar spatial-orientation hypothesis to account for certain Poggendorff results. Other single-factor explanations do worse. For instance, the converging-line display presents exactly the same "railroad track" depth feature as the Ponzo illusion regarded by Gregory (1968) as the prototype of visual distortions caused by misapplied constancy scaling.
But a misapplied-constancy explanation would seem to predict only underestimation of the angle between the line segments, a prediction clearly not compatible with the present results.

The new data also challenge the statement that acute angles tend to be overestimated. Underestimation of an acute angle was also demonstrated. There is, however, suspicion of another complicating factor. Among visual geometrical illusions of converging lines, the presence (as in the Poggendorff and Zöllner illusions) or absence (as in the Ponzo illusion) of an actually depicted intersection may be critical. Virsu's converging-line display is an impoverished version of the Poggendorff illusion. As yet unpublished data (Weintraub \& Krantz, 1969 ) indicate that for all angles less than $90 \mathrm{deg}$ between transversal and parallels, the Poggendorff illusory effects are invariably negative (the upper segment of the transversal is set too low when perceived collinearity is attained). Yet our asymmetrical converging line-segment illusion, with lines that do not intersect in the visual display, gives positive errors with small angles. A Poggendorff variant that replaced the parallels with a single nonintersecting vertical line (Fig. 2) with an angular separation of $11 \frac{11 / 4}{4} \mathrm{deg}$ gave a significant amount of positive illusion $(3.56 \mathrm{~mm})$, a finding at odds with the standard Poggendorff data.

An adequate single-factor explanation of converging-line illusions seems unlikely. With the very simple converging-line configuration employed, even the hypothesis of assimilation toward cardinal viewing axes leaves systematic variance unexplained. The hypothesis is, however, intuitively appealing, plausible, consistent with the work of others, and able to account handily for the most salient features of the new data.

\section{REFERENCES}

CHIANG, C. A new theory to explain geometrical illusions produced by crossing lines. Perception \& Psychophysics, 1968, 3 174-176.

GREEN, R. T., \& HOYLE, E. M. The influence of spatial orientation on the Poggendorff illusion. Acta Psychologica, 1964, 22 348-366.

GREGORY, R. L. Visual illusions. In B. M. Foss (Ed.), New horizons in psychology. Baltimore: Penguin Books, 1966. Pp. 68-96.

GREGORY, R. L. Visual illusions. Scientific American, 1968, 219, 66-76.

HOFMANN, F. B., \& BIELSCHOWSKY, A. Über die Einstellung der scheinbaren Horizontalen und Vertikalen bei Betrachtung eines von schrägen Konturen erfülten Gesichtsfeldes. Archiv der gesamten Physiologie, 1909, 126, 453-475.

HOTOPF, W. H. N. The size-constancy theory of visual illusions. British Journal of Psychology, $1966,57,307-318$.

LUCKIESH, M. Visual illusions: Their causes, characteristics and applications. New York: Dover, 1965.

RESTLE, F. Illusions of bent line. Perception \& Psychophysics, 1969, 5, 273-274.

von BÉKÉSY, G. Sensory inhibition. Princeton: Princeton University Press, 1967.

WALlaCE, G. K. Optical illusions. Nature, $1966,209,327-328$.

WEINTRAUB, D. J., \& KRANTZ, D. H. Much ado about the Poggendorff illusion. Paper presented at the meeting of the Psychonomic Society, St. Louis, November 1969.

WITKIN, H. A. The nature and importance of individual differences in perception. Journal of Personality, 1949, 18, 145-170.

\section{NOTE}

1. Rational reasons underlic the sequence of data collection. Phase 1 constituted a preliminary study. Phase 2 a full-fledged experiment. Precision was being sacrificed by ignoring Phase 1 data, so those data were retrieved with Phase 3 filling the gaps. Phase 4 was executed to determine more precisely the function for angular separations $<60 \mathrm{deg}$, specifically, to be certain that errors did not approach zero as angular separations approached zero. Statistical significance was never an issue, since, for any phase, points always differed significantly among themselves. Thus, the composite data of $1 \mathrm{ig} .3 \mathrm{~A}$ represent our best estimate concerning the form of the error function for symmetrical displays. Fach standard error in Fig. $3 \mathrm{~A}$ is based upon the total number of $O$ s judging an angular separation, dividing the estimated sigma by the square root of that total. Phase means are shown in lig. 3A as another reminder of the variability inherent in the data.

(Accepted for publication April 24, 1970.) 